\title{
Decorrelation of total mass via energy*
}

\author{
Le Chen \\ Davar Khoshnevisan \\ University of Utah \\ University of Utah \\ Kunwoo Kim \\ University of Utah
}

October 22, 2014

\begin{abstract}
The main result of this small note is a quantified version of the assertion that if $u$ and $v$ solve two nonlinear stochastic heat equations, and if the mutual energy between the initial states of the two stochastic PDEs is small, then the total masses of the two systems are nearly uncorrelated for a very long time. One of the consequences of this fact is that a stochastic heat equation with regular coefficients is a finite system if and only if the initial state is integrable.
\end{abstract}

Keywords. The stochastic heat equation; finite particle systems; total mass; mutual energy.

AMS 2010 subject classification. Primary 60H15, 60H25; Secondary 35R60, 60K37, 60J30, 60B15.

\section{Introduction}

Consider a stochastic partial differential equation of the type

$$
\frac{\partial}{\partial t} u_{t}(x)=\frac{\theta}{2} \frac{\partial^{2}}{\partial x^{2}} u_{t}(x)+\sigma\left(u_{t}(x)\right) \xi(t, x),
$$

for all $t>0$ and $x \in \mathbf{R}$, where $\xi$ denotes space-time white noise; that is, $\xi$ is a generalized centered Gaussian random field with covariance measure

$$
\operatorname{Cov}[\xi(t, x), \xi(s, y)]=\delta_{0}(s-t) \delta_{0}(x-y),
$$

for every $s, t \geqslant 0$ and $x, y \in \mathbf{R}$.

Throughout, the diffusion coeffficient $\theta / 2$ is assumed to be fixed, finite, and strictly positive. In order to keep the discussion as non-technical as possible

*Research supported in part by a Swiss Federal Fellowship (L.C.) and a grant from the United States' National Science Foundation (D.K.; DMS-1307470) 
we consider only the commonly-studied setting in which the initial function $u_{0}$ is nonrandom and essentially bounded, and the nonlinearity $\sigma: \mathbf{R} \rightarrow \mathbf{R}$ is nonrandom and globally Lipschitz continuous. The theory of Walsh [11] ensures the existence of a unique continuous solution $u$ to (1.1).

In addition, we assume throughout that $\sigma$ satisfies the following conditions:

$$
\sigma(x) \geqslant 0 \text { for all } x \in \mathbf{R} \text { and } \sigma(0)=0 .
$$

Since $\sigma$ vanishes at the origin and $u_{0}(x) \geqslant 0$ for all $x \in \mathbf{R}$, it follows from a comparison theorem $[8,9]$ that with probability one,

$$
u_{t}(x) \geqslant 0 \quad \text { for all } t \geqslant 0 \text { and } x \in \mathbf{R} .
$$

Because of this fact, and since $-\xi$ is also a space-time white noise, the positivity condition on $\sigma$ is harmless. Moreover, the positivity of $u$ suggests that we can think of $u_{t}(x)$ as the density at location $x$ of a continuous particle system at time $t$. The total mass process of that particle system at time $t \geqslant 0$ is therefore

$$
\int_{-\infty}^{\infty} u_{t}(x) \mathrm{d} x=\left\|u_{t}\right\|_{L^{1}(\mathbf{R})} .
$$

As a consequence, $t \mapsto\left\|u_{t}\right\|_{L^{1}(\mathbf{R})}$ is a continuous local martingale as long as it is finite at all times. This fact is basically due to Spitzer [10, Proposition 2.3] - see also the proof of Lemma 2.1 below - and plays an important role for example in Liggett's analysis of linear particle systems [7, Chapter IX].

Motivated by the preceding, one says [7, p. 432] that the system is finite if $\left\|u_{t}\right\|_{L^{1}(\mathbf{R})}<\infty$ a.s. for all $t \geqslant 0$ and that it is infinite if $\left\|u_{t}\right\|_{L^{1}(\mathbf{R})}=\infty$ a.s. for all $t \geqslant 0$. Because of the way in which we have defined things, it is logically possible that a particle system is neither finite nor infinite. The following shows that this sort of anomaly cannot occur in the context of (1.1). Moreover, that there is a very simple characterization of when (1.1) is a finite system.

Corollary 1.1. Let $u$ solve (1.1), starting with a nonrandom and nonnegative initial function $u_{0} \in L^{\infty}(\mathbf{R})$. Then, $u_{0} \in L^{1}(\mathbf{R})$ if and only if the system (1.1) is finite.

This result is stated as a corollary of an inequality [Theorem 2.3 below] that says roughly that if $u$ and $v$ solve (1.1) — with respective nonlinearities $\sigma_{1}$ and $\sigma_{2}$ and initial functions $u_{0}$ and $v_{0}$-and if the "mutual energy" between $u_{0}$ and $v_{0}$ is small, then the total mass of $u$ at time $t$ is almost uncorrelated from the total mass of $v$ at time $t$ for a wide range of times $t$. This fact has other interesting consequences as well. We name two of them next.

The following result says that if $u_{0}$ and $v_{0}$ have bounded support and the support of $u_{0}$ is very far away from the support of $v_{0}$, then the total mass of $u$ at time $t$ is almost uncorrelated from the total mass of $v$ at time $t$ for all large times $t$ upto a constant multiple of the distance between the supports of $u_{0}$ and $v_{0}$. In order to write this out more carefully, let $\mathcal{S}[f]$ denote the support of a function $f: \mathbf{R} \rightarrow \mathbf{R}$, and let "dist" denote the Hausdorff distance between 
subsets of the real line. Also, let $\operatorname{Lip}(\varphi)$ to be the optimal Lipschitz constant of a Lipschitz-continuous function $\varphi: \mathbf{R} \rightarrow \mathbf{R}$; that is,

$$
\operatorname{Lip}(\varphi):=\sup _{-\infty<a<b<\infty}\left|\frac{\varphi(a)-\varphi(b)}{a-b}\right| .
$$

Then the preceding takes the following more precise form.

Corollary 1.2. Suppose $u$ and $v$ solve (1.1) with respective nonrandom nonnegative initial functions $u_{0}, v_{0} \in L^{1}(\mathbf{R}) \cap L^{\infty}(\mathbf{R})$ that satisfy $\left\|u_{0}\right\|_{L^{1}(\mathbf{R})} \wedge$ $\left\|v_{0}\right\|_{L^{1}(\mathbf{R})}>0$ and diffusion coefficients $\sigma_{1}, \sigma_{2}$ that satisfy (1.3). Then there exist $\eta \in(0,1)$ and $\gamma>0$ - depending only on $\left(\operatorname{Lip}\left(\sigma_{1}\right), \operatorname{Lip}\left(\sigma_{2}\right), \theta\right)$ - such that

$$
\operatorname{Cov}\left(\frac{\left\|u_{t}\right\|_{L^{1}(\mathbf{R})}}{\left\|u_{0}\right\|_{L^{1}(\mathbf{R})}}, \frac{\left\|v_{t}\right\|_{L^{1}(\mathbf{R})}}{\left\|v_{0}\right\|_{L^{1}(\mathbf{R})}}\right) \leqslant \eta^{-1} \mathrm{e}^{-\gamma t}
$$

provided that $0<t<\eta \cdot \operatorname{dist}\left(\mathcal{S}\left[u_{0}\right], \mathcal{S}\left[v_{0}\right]\right)$.

In order to motivate our third corollary, we first digress a little. The Heisenberg uncertainty principle says, in one form or another, that a nice function $\varphi$ and its Fourier transform $\widehat{\varphi}$ cannot both have small support; see Donoho and Stark [5] for example. Therefore one expects that, for many nice pairs of functions $u_{0}$ and $v_{0}, \operatorname{dist}\left(\mathcal{S}\left[u_{0}\right], \mathcal{S}\left[v_{0}\right]\right)$ ought to be large if and only if the Lebesgue measure of $\mathcal{S}\left[\widehat{u}_{0}\right] \cap \mathcal{S}\left[\widehat{v}_{0}\right]$ is small. If this were true, then because of Corollary 1.2, one would also expect that if meas $\left(\mathcal{S}\left[\widehat{u}_{0}\right] \cap \mathcal{S}\left[\widehat{v}_{0}\right]\right)$ were small, then $\left\|u_{t}\right\|_{L^{1}(\mathbf{R})}$ and $\left\|v_{t}\right\|_{L^{1}(\mathbf{R})}$ have small covariance for a long time. The following shows that this last assertion is so, and also yields a quantitative estimate of the "how long."

Corollary 1.3. Suppose $u$ and $v$ solve (1.1) with respective nonrandom nonnegative initial functions $u_{0}, v_{0} \in L^{1}(\mathbf{R}) \cap L^{\infty}(\mathbf{R})$ and diffusion coefficients $\sigma_{1}$ and $\sigma_{2}$ respectively. Then there exist $\eta \in(0,1)$ and $\gamma>0$-depending only on $\operatorname{Lip}\left(\sigma_{1}\right), \operatorname{Lip}\left(\sigma_{2}\right)$, and $\theta$ - such that

$$
\operatorname{Cov}\left(\frac{\left\|u_{t}\right\|_{L^{1}(\mathbf{R})}}{\left\|u_{0}\right\|_{L^{1}(\mathbf{R})}}, \frac{\left\|v_{t}\right\|_{L^{1}(\mathbf{R})}}{\left\|v_{0}\right\|_{L^{1}(\mathbf{R})}}\right) \leqslant \eta^{-1} \mathrm{e}^{-\gamma t}
$$

provided that $0<t<\eta \cdot \log _{+}\left[1 / \operatorname{meas}\left(\mathcal{S}\left[\widehat{u}_{0}\right] \cap \mathcal{S}\left[\widehat{v}_{0}\right]\right)\right]$.

Let us conclude the Introduction with a brief comment on the previous corollary.

If the Fourier transforms of $u_{0}$ and $v_{0}$ were supported on disjoint sets then Corollary 1.3 would imply the extremely surprising statement that $\left\|u_{t}\right\|_{L^{1}(\mathbf{R})}$ and $\left\|v_{t}\right\|_{L^{1}(\mathbf{R})}$ are uncorrelated for all $t \geqslant 0$. It turns out that this condition of disjoint support typically does not hold for reasons that are similar to the reasons behind Heisenberg's uncertainty principle. In order to see this, let $A(\mathbf{R})$ denote the Wiener algebra; that is, the collection of all $f \in L^{1}(\mathbf{R})$ such that $\widehat{f} \in L^{1}(\mathbf{R})$. As usual, we identify two functions that are a.e. equal. Owing to the Riemann-Lebesgue lemma, $A(\mathbf{R}) \subset C_{0}(\mathbf{R})$ [the latter being the space 
of uniformly continuous functions on $\mathbf{R}$ that vanish at infinity]. In particular, $A(\mathbf{R})$ is dense in $L^{p}(\mathbf{R})$ for all $p \in(0, \infty]$; in turn, the Schwartz space $\mathscr{S}(\mathbf{R})$ of test functions of rapid decrease is dense in $A(\mathbf{R})$. To summarize, $A(\mathbf{R})$ is a big collection of real functions. Furthermore, the Fourier transform is a one-to-one and onto map from $A(\mathbf{R})$ to $A(\mathbf{R})$ thanks to the inversion theorem.

Now suppose that $u_{0}, v_{0} \in A(\mathbf{R})$ are nonnegative functions that have the additional property that their Fourier transforms have disjoint support. By the inversion theorem, $\widehat{u}_{0}$ and $\widehat{v}_{0}$ are both positive definite and continuous. They are, in particular, maximized at the origin [theorem of Herglotz]. This implies that at least one of $u_{0}$ and $v_{0}$ is identically zero.

It is possible to appeal to the works of Chen and Dalang [1] and Chen and Kim [2] in order to extend our results to the case that $u_{0}$ is a measure. For example, that extension of Corollary 1.1 asserts that the system is finite if and only if the initial measure is finite. See $\S 3.2$ below for more details.

\section{Some background, and an inequality}

We follow the theory of Walsh [11] - see in particular, Dalang [3] and Chen and Dalang [1] — and interpret the SPDE (1.1) as the random integral equation,

$$
u_{t}(x)=\left(p_{t} * u_{0}\right)(x)+(p \circledast \sigma(u))_{t}(x),
$$

where: (a) $(p \circledast \Psi)_{t}(x):=\int_{(0, t) \times \mathbf{R}} p_{t-s}(x-y) \Psi_{s}(y) \xi(\mathrm{d} s \mathrm{~d} y)$ is a stochastic integral, in the sense of Walsh [11], of any predictable random field $\Psi$ that satisfies $\int_{0}^{t} \mathrm{~d} s \int_{-\infty}^{\infty} \mathrm{d} y\left[p_{t-s}(y-x)\right]^{2} \mathrm{E}\left[\Psi_{s}(y)^{2}\right]<\infty$; and (b) $p$ denotes the heat kernel; i.e.,

$$
p_{r}(a):=(2 \pi \theta r)^{-1 / 2} \exp \left(-\frac{a^{2}}{2 \theta r}\right) \quad[r>0, a \in \mathbf{R}] .
$$

According to the theory of Dalang [3],

$$
\sup _{t \in[0, T]} \sup _{x \in \mathbf{R}} \mathrm{E}\left(\left|u_{t}(x)\right|^{k}\right)<\infty \quad \text { for all finite } T>0 \text { and } k \geqslant 2 .
$$

Moreover, the three quantities that appear naturally on both sides of (2.1) are continuous functions of $t>0$ and $x \in \mathbf{R}$ [up to a modification]. Therefore, there is a single null set off which (2.1) holds for all $t>0$ and $x \in \mathbf{R}$. We will refer to this fact tacitly from now on.

Let us mention an elementary consequence of Theorem 9 of Dalang and Mueller [4]. The following is well known to experts; we include the short proof for the sake of completeness.

Lemma 2.1. If $u_{0} \in L^{1}(\mathbf{R}) \cap L^{\infty}(\mathbf{R})$, then $u_{t} \in L^{1}(\mathbf{R})$ a.s. for all $t \geqslant 0$, and

$$
\sup _{t \in[0, T]} \mathrm{E}\left(\left\|u_{t}\right\|_{L^{1}(\mathbf{R})}^{2}\right)<\infty \quad \text { for all finite } T \geqslant 0 \text {. }
$$

In particular, $t \mapsto\left\|u_{t}\right\|_{L^{1}(\mathbf{R})}$ is a nonnegative continuous $L^{2}(\Omega)$-martingale. 
Proof. On one hand, Theorem 2.4 of Chen and Dalang [1] implies, as part of the definition of a solution, that

$$
\mathrm{E}\left(\int_{0}^{t} \mathrm{~d} s \int_{-\infty}^{\infty} \mathrm{d} y\left[\sigma\left(u_{s}(y)\right)\right]^{2}\right)<\infty
$$

for all $t>0$. On the other hand, the general theory of Walsh [11] implies that:

1. $t \mapsto \int_{(0, t) \times \mathbf{R}} \sigma\left(u_{s}(y)\right) \xi(\mathrm{d} s \mathrm{~d} y)$ defines a continuous $L^{2}$-martingale with quadratic variation $\int_{0}^{t} \mathrm{~d} s \int_{-\infty}^{\infty} \mathrm{d} y\left[\sigma\left(u_{s}(y)\right)\right]^{2}$ at time $t>0$; and

2. We have the stochastic Fubini theorem: Almost surely,

$$
\int_{-\infty}^{\infty}(p \circledast \sigma(u))_{t}(x) \mathrm{d} x=\int_{(0, t) \times \mathbf{R}} \sigma\left(u_{s}(y)\right) \xi(\mathrm{d} s \mathrm{~d} y) \quad \forall t>0 .
$$

The preceding allows us to integrate both sides of $(2.1)[\mathrm{d} x]$ in order to conclude that

$$
\left\|u_{t}\right\|_{L^{1}(\mathbf{R})}=\left\|u_{0}\right\|_{L^{1}(\mathbf{R})}+\int_{(0, t) \times \mathbf{R}} \sigma\left(u_{s}(y)\right) \xi(\mathrm{d} s \mathrm{~d} y) .
$$

The lemma follows. In fact, we also obtain the following estimate:

$$
\mathrm{E}\left(\left\|u_{t}\right\|_{L^{1}(\mathbf{R})}^{2}\right) \leqslant\left\|u_{0}\right\|_{L^{1}(\mathbf{R})}^{2}+\operatorname{Lip}^{2} \int_{0}^{t} \mathrm{E}\left(\left\|u_{s}\right\|_{L^{2}(\mathbf{R})}^{2}\right) \mathrm{d} s,
$$

valid for all $t>0$.

Lemma 2.2. Suppose $u$ and $v$ are the solutions to (1.1) with respect to diffusion coefficients $\sigma_{1}$ and $\sigma_{2}$ respectively, and nonrandom nonnegative initial functions $u_{0}, v_{0} \in L^{1}(\mathbf{R}) \cap L^{2}(\mathbf{R})$ respectively. Then,

$$
\operatorname{Cov}\left(\left\|u_{t}\right\|_{L^{1}(\mathbf{R})},\left\|v_{t}\right\|_{L^{1}(\mathbf{R})}\right)=\int_{0}^{t} \mathrm{~d} s \int_{-\infty}^{\infty} \mathrm{d} y \mathrm{E}\left[\sigma_{1}\left(u_{s}(y)\right) \sigma_{2}\left(v_{s}(y)\right)\right],
$$

for all $t>0$. Thus, $\left\|u_{t}\right\|_{L^{1}(\mathbf{R})}$ and $\left\|v_{t}\right\|_{L^{1}(\mathbf{R})}$ are positively correlated.

Proof. It follows from (2.1) that

$$
\begin{aligned}
\mathrm{E} & {\left[u_{t}(x) v_{t}\left(x^{\prime}\right)\right]=\left(p_{t} * u_{0}\right)(x)\left(p_{t} * v_{0}\right)\left(x^{\prime}\right) } \\
& +\int_{0}^{t} \mathrm{~d} s \int_{-\infty}^{\infty} \mathrm{d} y p_{t-s}(y-x) p_{t-s}\left(y-x^{\prime}\right) \mathrm{E}\left[\sigma_{1}\left(u_{s}(y)\right) \sigma_{2}\left(v_{s}(y)\right)\right] \mathrm{d} y,
\end{aligned}
$$

for all $t>0$ and $x, x^{\prime} \in \mathbf{R}$. Integrate $\left[\mathrm{d} x \mathrm{~d} x^{\prime}\right]$ to finish.

Before we continue our analysis of the covariance of the previous lemma, let us define the functions

$$
r_{\beta}(x):=\frac{1}{2 \sqrt{\beta \theta}} \exp (-|x| \sqrt{\beta / \theta}) \quad[\beta>0, x \in \mathbf{R}],
$$


and corresponding linear operators

$$
\left(\mathcal{R}_{\beta} f\right)(x):=\left(r_{\beta} * f\right)(x) \quad[\beta>0, x \in \mathbf{R}] .
$$

It is not hard to see that $\left\{\mathcal{R}_{\beta}\right\}_{\beta>0}$ is the resolvent of the heat semigroup for Brownian motion run at twice the standard speed. The corresponding Dirichlet forms are denoted by

$$
\mathcal{E}_{\beta}(f, g):=\left\langle f, \mathcal{R}_{\beta} g\right\rangle_{L^{2}(\mathbf{R})},
$$

and $\mathcal{E}_{\beta}(f, g)$ is called the mutual $\beta$-energy between $u_{0}$ and $v_{0}$, as is customary. The next theorem is the main result of this paper. Its content can be summarized loosely as follows: If $\mathcal{E}_{\beta}\left(u_{0}, v_{0}\right) \ll \exp (-\beta t)$ for some $t, \beta>0$, then $\left\|u_{s}\right\|_{L^{1}(\mathbf{R})}$ and $\left\|v_{s}\right\|_{L^{1}(\mathbf{R})}$ are nearly uncorrelated for all $s \in(0, t)$.

Theorem 2.3. For all $t \geqslant 0$ and $\beta>\left[\operatorname{Lip}\left(\sigma_{1}\right) \cdot \operatorname{Lip}\left(\sigma_{2}\right)\right]^{2} /(4 \theta)$,

$$
\operatorname{Cov}\left(\left\|u_{t}\right\|_{L^{1}(\mathbf{R})},\left\|v_{t}\right\|_{L^{1}(\mathbf{R})}\right) \leqslant \frac{2 \sqrt{\beta \theta} \operatorname{Lip}\left(\sigma_{1}\right) \operatorname{Lip}\left(\sigma_{2}\right)}{2 \sqrt{\beta \theta}-\operatorname{Lip}\left(\sigma_{1}\right) \operatorname{Lip}\left(\sigma_{2}\right)} \cdot \mathrm{e}^{\beta t} \mathcal{E}_{\beta}\left(u_{0}, v_{0}\right) .
$$

Proof. Because of (2.10) and the positivity assertions (1.3) and (1.4),

$$
\begin{aligned}
\mathrm{E}\left[u_{t}(x) v_{t}(x)\right] & \leqslant\left(p_{t} * u_{0}\right)(x)\left(p_{t} * v_{0}\right)(x) \\
& +\operatorname{Lip}\left(\sigma_{1}\right) \operatorname{Lip}\left(\sigma_{2}\right) \int_{0}^{t} \mathrm{~d} s \int_{-\infty}^{\infty} \mathrm{d} y\left[p_{t-s}(y-x)\right]^{2} \mathrm{E}\left[u_{s}(y) v_{s}(y)\right] \mathrm{d} y
\end{aligned}
$$

for every $t \geqslant 0$ and $x \in \mathbf{R}$. We integrate both side $[\mathrm{d} x]$ and appeal to the Tonelli theorem to find that

$$
\begin{aligned}
& \mathrm{E}\left[\left\langle u_{t}, v_{t}\right\rangle_{L^{2}(\mathbf{R})}\right] \\
& \leqslant\left\langle p_{t} * u_{0}, p_{t} * v_{0}\right\rangle_{L^{2}(\mathbf{R})}+\operatorname{Lip}\left(\sigma_{1}\right) \operatorname{Lip}\left(\sigma_{2}\right) \int_{0}^{t}\left\|p_{t-s}\right\|_{L^{2}(\mathbf{R})}^{2} \mathrm{E}\left[\left\langle u_{s}, v_{s}\right\rangle_{L^{2}(\mathbf{R})}\right] \mathrm{d} s,
\end{aligned}
$$

Since $p_{t} * p_{s}=p_{t+s}$ and $\left\langle p_{t} * f, g\right\rangle_{L^{2}(\mathbf{R})}=\left\langle f, p_{t} * g\right\rangle_{L^{2}(\mathbf{R})}$, this means that

$$
\begin{aligned}
& \mathrm{E}\left[\left\langle u_{t}, v_{t}\right\rangle_{L^{2}(\mathbf{R})}\right] \\
& \quad \leqslant\left\langle u_{0}, p_{2 t} * v_{0}\right\rangle_{L^{2}(\mathbf{R})}+\operatorname{Lip}\left(\sigma_{1}\right) \operatorname{Lip}\left(\sigma_{2}\right) \int_{0}^{t} p_{2(t-s)}(0) \mathrm{E}\left[\left\langle u_{s}, v_{s}\right\rangle_{L^{2}(\mathbf{R})}\right] \mathrm{d} s .
\end{aligned}
$$

Define, for all $\beta>0$,

$$
Z(\beta):=\int_{0}^{\infty} \mathrm{e}^{-\beta t} \mathrm{E}\left[\left\langle u_{t}, v_{t}\right\rangle_{L^{2}(\mathbf{R})}\right] \mathrm{d} t .
$$

Of course, $Z(\beta) \geqslant 0$ because of (1.4) and the Tonelli theorem. In addition, we can infer from (2.17) and Lemma 2.2 that

$$
\begin{aligned}
\operatorname{Cov}\left(\left\|u_{t}\right\|_{L^{1}(\mathbf{R})},\left\|v_{t}\right\|_{L^{1}(\mathbf{R})}\right) & \leqslant \operatorname{Lip}\left(\sigma_{1}\right) \operatorname{Lip}\left(\sigma_{2}\right) \cdot \int_{0}^{t} \mathrm{E}\left[\left\langle u_{s}, v_{s}\right\rangle_{L^{2}(\mathbf{R})}\right] \mathrm{d} s \\
& \leqslant \mathrm{e}^{\beta t} \operatorname{Lip}\left(\sigma_{1}\right) \operatorname{Lip}\left(\sigma_{2}\right) \cdot Z(\beta),
\end{aligned}
$$


valid for every $t, \beta>0$.

It is possible to see that $Z(\beta)<\infty$ for $\beta$ large. Here is a crude proof: Apply the Cauchy-Schwarz inequality twice in order to see that

$$
Z(\beta) \leqslant \int_{0}^{\infty} \mathrm{e}^{-\beta t} \sqrt{\mathrm{E}\left(\left\|u_{t}\right\|_{L^{2}(\mathbf{R})}^{2}\right) \cdot \mathrm{E}\left(\left\|v_{t}\right\|_{L^{2}(\mathbf{R})}^{2}\right)} \mathrm{d} t .
$$

As was shown in Foondun and Khoshnevisan [6], for every $\lambda>[\operatorname{Lip}(\sigma)]^{4} /(4 \theta)$ there exists a finite constant $C(\lambda)$ such that

$$
\mathrm{E}\left(\left\|u_{t}\right\|_{L^{2}(\mathbf{R})}^{2}\right) \leqslant C(\lambda) \mathrm{e}^{\lambda t} \quad \text { for all } t \geqslant 0 .
$$

Therefore,

$$
Z(\beta) \leqslant \sqrt{C\left(\lambda_{1}\right) \cdot C\left(\lambda_{2}\right)} \int_{0}^{\infty} \exp \left(-\left[\beta-\frac{\lambda_{1}+\lambda_{2}}{2}\right] t\right) \mathrm{d} t<\infty,
$$

for all positive $\lambda_{1}$ and $\lambda_{2}$ such that $\lambda_{j}>\left[\operatorname{Lip}\left(\sigma_{j}\right)\right]^{4} /(4 \theta)$ for $j=1,2$.

Consequently, we can deduce from (2.17) that as long as $Z(\beta)<\infty$-and this happens for all $\beta$ sufficiently large - we have

$$
\begin{aligned}
Z(\beta) & \leqslant \int_{0}^{\infty} \mathrm{e}^{-\beta t}\left\langle u_{0}, p_{2 t} * v_{0}\right\rangle_{L^{2}(\mathbf{R})} \mathrm{d} t+\operatorname{Lip}\left(\sigma_{1}\right) \operatorname{Lip}\left(\sigma_{2}\right) r_{\beta}(0) Z(\beta) \\
& =\mathcal{E}_{\beta}\left(u_{0}, v_{0}\right)+\operatorname{Lip}\left(\sigma_{1}\right) \operatorname{Lip}\left(\sigma_{2}\right) r_{\beta}(0) Z(\beta),
\end{aligned}
$$

since $r_{\beta}(x):=\int_{0}^{\infty} \mathrm{e}^{-\beta t} p_{2 t}(x) \mathrm{d} t$, as can be seen directly from (2.11).

Thus, whenever $Z(\beta)<\infty$, we have

$$
Z(\beta) \leqslant \mathcal{E}_{\beta}\left(u_{0}, v_{0}\right)\left[1-\frac{\operatorname{Lip}\left(\sigma_{1}\right) \operatorname{Lip}\left(\sigma_{2}\right)}{2 \sqrt{\beta \theta}}\right]^{-1} .
$$

An elementary iteration of (2.17) shows that the preceding holds, in fact, whenever it makes sense [that is, whenever the right-hand side is strictly positive]. Equivalently, that $(2.23)$ is valid for all $\beta>(4 \theta)^{-1}\left[\operatorname{Lip}\left(\sigma_{1}\right) \cdot \operatorname{Lip}\left(\sigma_{2}\right)\right]^{2}$. The result follows from (2.19).

\section{Proof of the corollaries}

Armed with Theorem 2.3 we conclude the paper by establishing its corollaries.

\subsection{Proof of Corollary 1.1}

If $u_{0} \in L^{1}(\mathbf{R})$, then $u_{t}$ is a.s. integrable for all $t>0$; see for example Lemma 2.1. We work on the more interesting converse next.

Suppose $u_{0} \in L^{\infty}(\mathbf{R})$ is not in $L^{1}(\mathbf{R})$; specifically, we are assuming that

$$
\left\|u_{0}\right\|_{L^{1}(\mathbf{R})}=\int_{-\infty}^{\infty} u_{0}(x) \mathrm{d} x=\infty .
$$


Define

$$
u_{0, N}(x):=u_{0}(x) \mathbf{1}_{[-N, N]}(x) \quad[x \in \mathbf{R}, N=1,2, \ldots],
$$

and let $u_{t, N}(x)$ denote the solution to (1.1), starting from initial function $u_{0, N}$; that is,

$$
u_{t, N}(x):=\left(p_{t} * u_{0, N}\right)(x)+\int_{(0, t) \times \mathbf{R}} p_{t-s}(y-x) \sigma\left(u_{s, N}(y)\right) \xi(\mathrm{d} s \mathrm{~d} y) .
$$

Since $u_{0}(x) \geqslant u_{0, N}(x)$ for all $x \in \mathbf{R}$ and $N \geqslant 1$, an appeal to the comparison theorem of SPDEs $[8,9]$ shows that $u_{t}(x) \geqslant u_{t, N}(x)$ for all $t \geqslant 0, x \in \mathbf{R}$, and $N \geqslant 1$, all off a single P-null set. In particular,

$$
\left\|u_{t}\right\|_{L^{1}(\mathbf{R})} \geqslant\left\|u_{t, N}\right\|_{L^{1}(\mathbf{R})} \quad \text { for all } t \geqslant 0 \text { and } N \geqslant 1,
$$

P-almost surely. It remains to prove that

$$
\limsup _{N \rightarrow \infty}\left\|u_{t, N}\right\|_{L^{1}(\mathbf{R})}=\infty \quad \text { a.s. }
$$

Because of (1.4), $u_{t, N}(x) \geqslant 0$ for all $t \geqslant 0, N \geqslant 1$, and $x \in \mathbf{R}$ a.s. Therefore, $\left\|u_{t, N}\right\|_{L^{1}(\mathbf{R})}=\int_{-\infty}^{\infty} u_{t, N}(x) \mathrm{d} x$. In particular, (3.1), (3.3), and the monotone convergence theorem together yield

$$
\lim _{N \rightarrow \infty} \mathrm{E}\left(\left\|u_{t, N}\right\|_{L^{1}(\mathbf{R})}\right)=\lim _{N \rightarrow \infty}\left\|u_{0, N}\right\|_{L^{1}(\mathbf{R})}=\infty .
$$

By Chebyshev's inequality, we obtain (3.5) - and hence the result-once we prove that for every $t \geqslant 0$ there exists a finite constant $K(t)$ such that

$$
\operatorname{Var}\left(\left\|u_{t, N}\right\|_{L^{1}(\mathbf{R})}\right) \leqslant K(t) \cdot \mathrm{E}\left(\left\|u_{t, N}\right\|_{L^{1}(\mathbf{R})}\right) \quad \text { for all } N \geqslant 1 .
$$

According to Theorem 2.3, there exist finite constants $A>0$ and $\beta>0$ such that for all $t \geqslant 0$ and $N \geqslant 1$,

$$
\begin{aligned}
\operatorname{Var}\left(\left\|u_{t, N}\right\|_{L^{1}(\mathbf{R})}\right) & \leqslant A \mathrm{e}^{\beta t} \cdot \int_{-\infty}^{\infty} u_{0, N}(x)\left(\mathcal{R}_{\beta} u_{0, N}\right)(x) \mathrm{d} x \\
& \leqslant A \mathrm{e}^{\beta t} \cdot \sup _{x \in \mathbf{R}}\left(\mathcal{R}_{\beta} u_{0}\right)(x) \cdot \mathrm{E}\left(\left\|u_{t, N}\right\|_{L^{1}(\mathbf{R})}\right),
\end{aligned}
$$

because $u_{0, N} \leqslant u_{0}$ whence $\mathcal{R}_{\beta} u_{0, N} \leqslant \mathcal{R}_{\beta} u_{0}$; eq. (3.6) justifies the last line. Thus follows (3.7), since

$$
\sup _{y \in \mathbf{R}}\left(\mathcal{R}_{\beta} u_{0}\right)(y) \leqslant\left\|u_{0}\right\|_{L^{\infty}(\mathbf{R})} \cdot \int_{-\infty}^{\infty} r_{\beta}(x) \mathrm{d} x=\beta^{-1}\left\|u_{0}\right\|_{L^{\infty}(\mathbf{R})} .
$$

The integral was evaluated directly from (2.11). 


\subsection{Remarks on initial measures}

As we mentioned in the Introduction, the results of this paper extend to the case that $u_{0}$ is a measure without a great deal of extra effort. Let us point out how this is done for Corollary 1.1.

Corollary 3.1. Let $u$ solve (1.1) for all $x \in \mathbf{R}$, starting from a nonrandom Borel measure $u_{0}$ on $\mathbf{R}$ that satisfies

$$
\int_{-\infty}^{\infty} \mathrm{e}^{-c x^{2}} u_{0}(\mathrm{~d} x)<\infty \quad \text { for all } c>0 .
$$

Then $u_{0}$ is finite iff the system (1.1) is finite.

Sketch of Proof. The proof is basically an adaptation of the proof of Corollary 1.1 to the case that $u_{0}$ is a measure. We hash out the details only where they are not standard.

Theorem 2.4 of Chen and Dalang [1] tells us that, under Condition (3.10), (1.1) has a solution $u$ that is uniquely defined by the following moment condition: $\sup _{x \in \mathbf{R}} \mathrm{E}\left(\left|u_{t}(x)\right|^{k}\right)<\infty$ for all $t>0$ and $k \geqslant 1$. Standard arguments then imply that the process $\left\{u_{t}\right\}_{t \geqslant 0}$ is Markov. We now condition at time $t$ and appeal to the preceding moment condition, together with the well-known fact that if we start (1.1) from an independent, $L^{k}(\Omega)$-bounded initial function, then the solution is Hölder continuous. It follows that $u$ is Hölder continuous a.s. at all points $(t, x)$ where $t>0$ and $x \in \mathbf{R}$. This comment will take care of all of the meaurability issues that might crop up in the remainder of the proof.

If $u_{0}$ has finite total mass, then Theorem 2.4 of [1] and Lemma 2.1 together imply that $\left\|u_{t}\right\|_{L^{1}(\mathbf{R})}<\infty$ a.s. for all $t>0$ in exactly the same way that the corresponding result held for $u_{0} \in L^{1}(\mathbf{R}) \cap L^{\infty}(\mathbf{R})$. Let us, therefore, consider the more interesting case that $u_{0}(\mathbf{R})=\infty$; of course, (3.10) is still in place. We propose to prove that $\left\|u_{t}\right\|_{L^{1}(\mathbf{R})}=\infty$ a.s. for all $t>0$.

For every Borel set $A \subseteq \mathbf{R}$ and all integers $n$ define

$$
v_{0}(A \cap[n, n+1)):=\frac{u_{0}(A \cap[n, n+1))}{u_{0}([n, n+1)) \vee 1} .
$$

This construction uniquely describes a Borel measure $v_{0}$ on $\mathbf{R}$ that is dominated by $u_{0}$ in the sense that $v_{0}(A) \leqslant u_{0}(A)$ for all linear Borel sets $A$. In particular, $v_{0}$ satisfies $(3.10)$. We observe also that $v_{0}$ is an infinite measure.

Let $v_{t}(x)$ denote the solution to (1.1), started at measure $v_{0}$. The comparison theorem of Chen and $\operatorname{Kim}[2]$ shows that $v_{t}(x) \leqslant u_{t}(x)$ for all $x \in \mathbf{R}$ and $t>0$ a.s., and hence $\left\|v_{t}\right\|_{L^{1}(\mathbf{R})} \leqslant\left\|u_{t}\right\|_{L^{1}(\mathbf{R})}$. In order to complete our proof we propose to show that $\left\|v_{t}\right\|_{L^{1}(\mathbf{R})}=\infty$ a.s. for all $t>0$.

Since $v_{0}([n, n+1)) \leqslant 1$ and $\sum_{n=-\infty}^{\infty} r_{\beta}(n) \leqslant r_{\beta}(0)+2 \int_{0}^{\infty} r_{\beta}(x) \mathrm{d} x=$ $(4 \beta \theta)^{-1 / 2}+2 / \beta$, the convexity of $x \mapsto|x|$ shows that for all $\beta>0$,

$$
\left(\mathcal{R}_{\beta} v_{0}\right)(x) \leqslant \sum_{n=-\infty}^{\infty} \max _{y \in[n, n+1]} r_{\beta}(y-x) \leqslant(4 \beta \theta)^{-1 / 2}+4 \beta^{-1}
$$


uniformly for all $x \in \mathbf{R}$. In other words, $v_{0}$ is an infinite measure that has a bounded $\beta$-potential for every $\beta>0$; compare with (3.9). From here on, we can mimic our derivation of Corollary 1.1 - starting with $v_{0, N}:=$ the restriction of $v_{0}$ to $[-N, N]$-in order to deduce that $\left\|v_{t}\right\|_{L^{1}(\mathbf{R})}=\infty$ a.s. for all $t>0$. We omit the remaining details.

\subsection{Proof of Corollary 1.2}

Let $\Delta$ denote the Hausdorff distance between the supports of $u_{0}$ and $v_{0}$. If $\Delta=0$ then the result holds vacuously. Therefore, we will consider only the case that $\Delta>0$. In that case,

$$
\begin{aligned}
\mathcal{E}_{\beta}\left(u_{0}, v_{0}\right) & =\frac{1}{2 \sqrt{\beta \theta}} \int_{-\infty}^{\infty} \mathrm{d} x \int_{-\infty}^{\infty} \mathrm{d} y \mathrm{e}^{-|x-y| \sqrt{\beta / \theta}} u_{0}(x) v_{0}(y) \\
& \leqslant \frac{1}{2 \sqrt{\beta \theta}}\left\|u_{0}\right\|_{L^{1}(\mathbf{R})}\left\|v_{0}\right\|_{L^{1}(\mathbf{R})} \cdot \mathrm{e}^{-\Delta \sqrt{\beta / \theta}} .
\end{aligned}
$$

Consequently, Theorem 2.3 assures us that simultaneously for all $t \geqslant 0$ and $\beta>\left[\operatorname{Lip}\left(\sigma_{1}\right) \cdot \operatorname{Lip}\left(\sigma_{2}\right)\right]^{2} /(4 \theta)$,

$$
\operatorname{Cov}\left(\left\|u_{t}\right\|_{L^{1}(\mathbf{R})},\left\|v_{t}\right\|_{L^{1}(\mathbf{R})}\right) \leqslant \frac{K \exp (\beta t-\Delta \sqrt{\beta / \theta})}{2 \sqrt{\beta \theta}-\operatorname{Lip}\left(\sigma_{1}\right) \operatorname{Lip}\left(\sigma_{2}\right)} .
$$

where $K:=\operatorname{Lip}\left(\sigma_{1}\right) \operatorname{Lip}\left(\sigma_{2}\right) \cdot\left\|u_{0}\right\|_{L^{1}(\mathbf{R})}\left\|v_{0}\right\|_{L^{1}(\mathbf{R})}$. Now choose $\beta:=\delta(\Delta / t)^{2}$ for a suitable choice of $\delta>0$ in order to finish.

\subsection{Proof of Corollary 1.3}

By Parseval's identity,

$$
\begin{aligned}
& \mathcal{E}_{\beta}\left(u_{0}, v_{0}\right)=\frac{1}{2 \pi} \int_{-\infty}^{\infty} \widehat{u}_{0}(z) \overline{\widehat{v}_{0}(z)} \widehat{r}_{\beta}(z) \mathrm{d} z=\frac{1}{2 \pi} \int_{-\infty}^{\infty} \frac{\widehat{u}_{0}(z) \overline{\widehat{v}_{0}(z)}}{\beta+\theta z^{2}} \mathrm{~d} z \\
& \leqslant \frac{1}{2 \pi \beta} \int_{-\infty}^{\infty}\left|\widehat{u}_{0}(z) \widehat{v}_{0}(z)\right| \mathrm{d} z \\
& \leqslant \frac{1}{2 \pi \beta}\left\|u_{0}\right\|_{L^{1}(\mathbf{R})}\left\|v_{0}\right\|_{L^{1}(\mathbf{R})} \cdot \operatorname{meas}\left(\mathcal{S}\left[\widehat{u}_{0}\right] \cap \mathcal{S}\left[\widehat{v}_{0}\right]\right),
\end{aligned}
$$

since the Fourier transform of every function $\varphi \in L^{1}(\mathbf{R})$ is bounded uniformly in modulus by $\|\varphi\|_{L^{1}(\mathbf{R})}$. The corollary follows easily from this bound. The remainder of the proof is similar to that of Corollary 1.2, and hence omitted.

\section{References}

[1] Chen, Le and Robert C. Dalang. Moments and growth indices for nonlinear stochastic heat equation with rough initial conditions. To appear in Ann. Probab. Preprint available at arXiv:1307.0600 (2014). 
[2] Chen, Le and Kunwoo Kim. On comparison principle and strict positivity of solutions to the nonlinear stochastic fractional heat equations. Submitted for publication. Preprint available at arXiv:1410.0604 (2014).

[3] Dalang, Robert C. Extending the martingale measure stochastic integral with applications to spatially homogeneous s.p.d.e.'s. Electron. J. Probab. 4(6) (1999) 29 pp. (electronic).

[4] Dalang, Robert C. and Carl Mueller. Some non-linear S.P.D.E.'s that are second order in time. Electron. J. Probab. 8(1) (2003) 21 pp. (electronic).

[5] Donoho, David L. and Philip B. Stark. Uncertainty principles and signal recovery. SIAM J. Appl. Math. 49(3) (1989) 906-931.

[6] Foondun, Mohammud and Davar Khoshnevisan. On the global maximum of the solution to a stochastic heat equation with compact-support initial data. Ann. Inst. Henri Poincaré Probab. Stat. 46(4) (2010) 895-907.

[7] Liggett, T. M. Interacting Particle Systems. Springer-Verlag, New York, 1985.

[8] Mueller, Carl. Some Tools and Results for Parabolic Stochastic Partial Differential Equations (English summary). In: A Minicourse on Stochastic Partial Differential Equations, 111-144, Lecture Notes in Math. 1962 Springer, Berlin, 2009.

[9] Mueller, Carl. On the support of solutions to the heat equation with noise. Stoch. E Stoch. Rep. 37(4) (1991) 225-245.

[10] Spitzer, Frank. Infinite systems with locally interacting components. Ann. Probab. 9 (1981) 349-364.

[11] Walsh, John B. An Introduction to Stochastic Partial Differential Equations. In: Ėcole d'èté de probabilités de Saint-Flour, XIV-1984, 265-439. Lecture Notes in Math. 1180 Springer, Berlin, 1986.

Le Chen, Davar Khoshnevisan, and Kunwoo Kim

Department of Mathematics, University of Utah, Salt Lake City, UT 84112-0090

Emails \& URLs:

chen@math . utah.edu

http: //www . math. utah.edu/ chen/

davar@math . utah.edu

http://www. math. utah.edu/ davar/

kkim@math.utah.edu

http://www.math.utah.edu/ kkim/ 